\title{
A SYSTEMATIC REVIEW OF SCIENTIFIC STUDIES ON THE EFFECTS OF MUSIC IN PEOPLE WITH OR AT RISK FOR EATING DISORDERS
}

\author{
Francesca Testa $^{1,2}$, Sarah Arunachalam ${ }^{2}$, Annie Heiderscheit ${ }^{3}$ \& Hubertus Himmerich ${ }^{1,4}$ \\ ${ }^{1}$ Department of Psychological Medicine, Institute of Psychiatry, Psychology \& Neuroscience, King's College London, \\ London, UK \\ ${ }^{2}$ Faculty of Life Sciences and Medicine, King's College London, London, United Kingdom \\ ${ }^{3}$ Department of Music Therapy, Augsburg University, Minneapolis, MN, USA \\ ${ }^{4}$ South London and Maudsley NHS Foundation Trust, London, UK
}

received: 25.10.2019;

revised: 3.3.2020;

accepted: 17.3 .2020

\section{SUMMARY}

Background: The prevalence of the three main eating disorders (EDs) anorexia nervosa (AN), bulimia nervosa (BN) and binge eating disorder (BED) is increasing, and a growing number of patients with EDs is seeking professional help. Thus, there is a need for additional treatment strategies in EDs. The aim of this review was to summarize the literature on the benefits and risks of music as well as the evidence for its therapeutic application in people with EDs.

Methods: Following the PRISMA guidelines, we performed a systematic literature review on scientific studies on the effect of music in people with or at risk for EDs using PubMed and the Web of Science database. The search terms used were: "music", "music therapy", "eating disorders", "anorexia nervosa”, "bulimia nervosa” and "binge eating disorder".

Results: 16 out of 119 identified and screened articles qualified as scientific studies involving a total of 3,792 participants. They reported on the use of music or music therapy in individuals with or at risk of AN and BN, but not BED. In inpatients with AN, listening to classical music was beneficial to food consumption. Singing in a group reduced post-prandial anxiety in AN inpatients and outpatients. Vodcasts which also included positive visual or autobiographical stimuli helped BN patients with anxiety and body image perception. Songwriting and sessions with a Body Monochord helped with the processing of therapeutically relevant topics in AN. Watching music videos, however, reinforced body dissatisfaction, drive for thinness, bodyweight concerns, preoccupation with physical appearance in pre-teenage and teenage girls, and drive for muscularity in adolescent boys.

Conclusions: These findings suggest that the therapeutic application of music may be beneficial in patients with AN and BN. However, the availability of studies with a rigorous randomized controlled trial (RCT) design is scarce.

Key words: music - eating disorders - anorexia nervosa - bulimia nervosa - binge eating disorder

\section{INTRODUCTION}

Eating disorders (EDs) represent a world-wide cause of psychiatric and physical morbidity and mortality. The three most widely recognized and researched EDs are anorexia nervosa (AN), bulimia nervosa (BN) and binge eating disorder (BED) (American Psychiatric Association 2013). The overall incidence of EDs has been reported to have significantly increased over the last decade (Darby et al. 2009, Micali et al. 2013, Smink et al. 2012, Schmidt et al. 2016). This increase is reflected in the rising numbers of people with EDs presenting to health services. Moreover, the illness is affecting people at an increasingly younger age (Smink et al. 2012, Schmidt et al. 2016).

The prevalence of AN is estimated to be about $1 \%$ among women, with a male to female ratio of approximately 1:10 (Keski-Rahkonen \& Mustelin 2016), and BN occurs in $1-2 \%$ of women with a male to female ratio of about 1:10 (Keski-Rahkonen \& Mustelin 2016). EDs are associated with significantly elevated mortality (Himmerich et al. 2019a, Himmerich et al. 2019b).

According to the Diagnostic and Statistical Manual of Mental Disorders (DSM-5), AN is characterized by significantly low body weight in the context of age, sex, and physical health; an intense fear of weight gain and disturbed body perception (American Psychiatric Association 2013). In the International Classification of Diseases-10 (ICD-10), amenorrhea is an additional diagnostic criterium (World Health Organization 1992), although it does not apply to male patients or female patients on oral contraceptives. The diagnostic criteria for BN are consistent across the ICD-10 and DSM-5; they encompass recurrent binge eating with compensatory behaviors and morbid preoccupation regarding body image and shape. BED is not listed in the ICD-10 but constitutes a diagnosis in the DSM-5. Here, it consists of recurrent episodes of binge eating along with the feeling of loss of control over the amount and type of food eaten (American Psychiatric Association 2013). BED is associated with feelings of embarrassment, guilt, and disgust, and often co-exists with affective or anxiety disorders. Unlike $\mathrm{BN}$, there are no compensatory behaviors pre-binge or postbinge. However, there is a considerable diagnostic flux within the spectrum of eating disorders (Milos et al. 2005). 
Treatment options for AN aim to address the primary features and symptoms of the disease - weight, appetite, distorted perceptions and behaviors, as well as secondary problems associated with mood and anxiety (Bodell \& Keel 2010). According to the guidelines by the National Institute for Health and Care Excellence (NICE), treatment for AN should take place in contact with or be provided by specialist services. Furthermore, it should include psychoeducation, monitoring of weight, mental and physical health, and risk factors, be multidisciplinary and coordinated between services, and involve the person's family members or carers (National Institute for Health and Care Excellence 2017). Psychological therapies are available, although with limited effectiveness. Recommended psychological therapies with scientific evidence proving effectiveness include individual Eating-Disorder-focused Cognitive Behavioural Therapy (CBT-ED), Maudsley Anorexia Nervosa Treatment for Adults (MANTRA) and Specialist Supportive Clinical Management (SSCM) (National Institute for Health and Care Excellence 2017). Pharmacological treatment approaches for AN consist mainly of weightinducing antipsychotics like olanzapine. Unfortunately, due to a lack of convincing randomized controlled trials (RCTs), there is no approved drug treatment available for patients with AN (Himmerich \& Treasure 2018).

The management of BN is mainly outpatient-based. According to NICE, BN-focused guided self-help should be considered first. However, most scientific evidence supports treatment with individual CBT-ED (Slade et al. 2018). The main aim of treatment for $B N$ is to disrupt the vicious cycle of over-eating and vomiting and to understand the triggers (Rushing et al. 2003). BNfocused Family Therapy (FT-BN) should be offered to children and young people with BN (National Institute for Health and Care Excellence 2017). Pharmacological therapy with fluoxetine in adjunct to CBT has shown to be superior to psychological therapy alone in improving symptoms of BN (Rushing et al. 2003). Fluoxetine is approved for the treatment of AN in most countries. Additionally, several RCTs show that topiramate effectively reduces binge eating and self-induced vomiting in BN while being well-tolerated (Himmerich \& Treasure 2018).

BED is often associated with high body weight, as a result of overeating with no compensatory mechanism. Hence, treatment aims to address the urge to binge as well as weight loss. Currently, binge eating is primarily managed with psychological therapy, either CBT or structured self-help (Linardon et al. 2017, Vocks et al. 2010). Lisdexamfetamine (LDX), a pro-drug of amphetamine, is the only drug approved by the Food and Drug Administration (FDA) for the treatment of moderate to severe BED in adults. This treatment is only approved in the US; its use in other countries is off-label (Himmerich \& Treasure 2018).

Table 1 provides an overview of prevalence, diagnostic criteria and currently discussed psychological and psychopharmacological treatment.
Among the ED spectrum, AN is associated with the lowest recovery rate (Herzog et al. 1993). An extensive review of the clinical outcomes of AN highlighted full recovery in less than $50 \%$ of the patients, improvement in 33\% and chronicity in 20\% (Steinhausen 2009). BN is associated with a better prognosis. Data showed that over $50 \%$ of individuals diagnosed with BN fully recover, 20\% remain chronically ill and 30\% relapse into symptoms of BN (Keel \& Mitchell 1997). Current treatment outcomes for all EDs are poor. Outpatient interventions usually fail to achieve weight restoration in the vast majority of AN patients (Guarda 2008), and only about $30 \%$ of patients with AN recover after 9 years (Himmerich \& Treasure 2018, Eddy et al. 2017). Therefore, there is an urgent need for therapeutic improvement.

Active and receptive music therapy (MT), group and individual MT have been applied in patients with EDs. The scientific literature on MT in EDs, mainly consisting of case reports and therapists' experiences, highlights that music can help patients with an ED discover their authentic self, support their ability to reclaim their life from the disorder, build resilience, work through issues of sexual identity, reinforce psychotherapy, provide means of expression and understanding beyond words, help to develop a positive self-esteem, developing healthy coping skills, and foster a sense of empowerment (Heiderscheit 2016). However, the empirical evidence from controlled clinical trials for the use of MT in EDs is limited, even though the literature is robust with clinical case illustrations; for a comprehensive review and further literature see (Heiderscheit 2016).

Many people with eating disorders suffer from other psychiatric comorbidities such as affective disorders (e.g. depression), anxiety disorders and obsessive-compulsive disorder (OCD) (Blinder et al. 2006, Spindler \& Milos 2007). In these disorders, music has been applied as a therapeutic strategy. In depression, MT used as an adjunct to pharmacological and psychological therapy improved depressive symptoms compared to standard interventions alone (Aalbers et al. 2017, Heise et al. 2013). Similarly, the application of MT to standard medical treatment was more effective in improving anxiety symptomatology in generalized anxiety disorder (GAD) (Gutiérrez \& Camarena 2015) and in reducing obsessive thoughts in OCD (Shiranibidabadi \& Mehryar 2015). Although NICE guidelines strongly recommend a multidisciplinary approach in the management of EDs, they do not specifically mention MT as a treatment option (National Institute for Health and Care Excellence 2017).

Music has been shown to affect different brain areas which play a significant role in many psychiatric disorders, with passive listening activating both subcortical (such as the medial geniculate body in the thalamus and the amygdala) and cortical areas (such as the auditory cortex) and active participation stimulating the basal ganglia, cerebellum and cortical motor area (Yinger \& Gooding 2014). 
Table 1. Prevalence, diagnostic criteria, psychological and psychopharmacological treatment according to Himmerich \& Treasure (Himmerich \& Treasure 2018)

\begin{tabular}{|c|c|c|c|c|}
\hline Eating disorder & Prevalence & Diagnostic criteria & Psychological treatment & $\begin{array}{l}\text { Psychopharmacological } \\
\text { treatment }\end{array}$ \\
\hline $\begin{array}{l}\text { Anorexia } \\
\text { nervosa }\end{array}$ & $\begin{array}{l}\text { - } \sim 1 \% \text { among } \\
\text { women } \\
\text { - Sex ratio men/ } \\
\text { women: } 1 / 10\end{array}$ & $\begin{array}{l}\text { - Significantly low body } \\
\text { weight } \\
\text { - Intense fear of weight gain } \\
\text { - Disturbed body perception }\end{array}$ & $\begin{array}{l}\text { - Eating-Disorder-focused } \\
\text { Cognitive Behavioral } \\
\text { Therapy (CBT-ED) } \\
\text { - Maudsley Anorexia } \\
\text { Nervosa Treatment for } \\
\text { Adults (MANTRA) } \\
\text { - Specialist Supportive } \\
\text { Clinical Management } \\
\text { (SSCM). }\end{array}$ & $\begin{array}{l}\text { Currently tested but not } \\
\text { approved medications: } \\
\text { - Olanzapine } \\
\text { - Aripiprazole } \\
\text { - Dronabinol }\end{array}$ \\
\hline Bulimia nervosa & $\begin{array}{l}\text { - } 1-2 \% \text { among } \\
\text { women } \\
\text { - Sex ratio: } 1 / 10\end{array}$ & $\begin{array}{l}\text { - Recurrent binges } \\
\text { - Compensatory behaviors } \\
\text { - Self-evaluation unduly } \\
\text { influenced by body shape } \\
\text { and weight }\end{array}$ & $\begin{array}{l}\text { - Bulimia-nervosa-focused } \\
\text { guided self-help } \\
\text { - CBT-ED } \\
\text { - Bulimia-Nervosa- } \\
\text { focused Family Therapy } \\
\text { (FT-BN; } \\
\text { in children) }\end{array}$ & $\begin{array}{l}\text { - Fluoxetine } \\
\text { - Topiramate } \\
\text { (not approved) }\end{array}$ \\
\hline $\begin{array}{l}\text { Binge eating } \\
\text { disorder }\end{array}$ & $\begin{array}{l}\text { - } 3 \% \text { of the } \\
\text { population } \\
\text { - Sex ratio } 1 / 3\end{array}$ & $\begin{array}{l}\text { - Binge eating } \\
\text { - No extreme compensatory } \\
\text { strategies }\end{array}$ & $\begin{array}{l}\text { - Structured self-help } \\
\text { - CBT }\end{array}$ & $\begin{array}{l}\text { - Lisdexamfetamine } \\
\text { (approved in } \\
\text { the USA only) }\end{array}$ \\
\hline
\end{tabular}

With this review, we sought to summarize the published benefits and potential side effects of the use of music as well as the evidence for its therapeutic application in people with EDs.

\section{METHODS}

This systematic review was conducted on 29/01/2020 following the recommendations outlined in the PRISMA guidelines (Moher et al. 2009).

\section{Selection Criteria}

We screened articles reporting studies of any design that assessed the use of music or MT on people with EDs.

Articles were included if:

- They described studies

- Music was part of the experimental or observational study design

- Measurable results or outcomes were reported Articles were excluded if:

- Music was not applied

- Measurable outcomes or effects were not reported

- The study dealt mainly with dance therapy

- The article was not an original publication (e.g. review, case report, meeting abstract, book review)

- The article dealt mainly with the mental health of musicians, music students, and music therapists

- The article reports animal studies

- The article focusses on the description of a hospital or a therapy program

- The article was not written in English.

\section{Search Strategy}

For the literature search, we used the medical database PubMed and the scientific database Web of Science comprising Web of Science Core Collection, BIOSIS Citation Index, KCI-Korean Journal Database, MEDLINE ${ }^{\circledR}$, Russian Science Citation Index and SciELO Citation Index. The search was performed from inception until the 29/01/2020 using the following key search terms in PubMed: (("music"[Title/Abstract]) OR ("music therapy"[Title/Abstract])) AND (("eating disorders"[Title/ Abstract]) OR ("anorexia nervosa"[Title/Abstract]) OR ("anorexia"[Title/Abstract]) OR ("bulimia nervosa"[Title/ Abstract]) OR ("bulimia "[Title/Abstract]) OR ("binge eating" [Title/Abstract]) OR ("binge eating disorder"[Title/Abstract])). In Web of Science, we used search terms: $\mathrm{TS}=$ (music OR music therapy) AND TS= (eating disorders OR anorexia nervosa OR anorexia OR bulimia nervosa OR bulimia OR binge eating OR binge eating disorder).

This search was supplemented by internet searches, hand-searches of reference lists in included papers and potentially relevant reviews. The eligible articles were further reviewed in full text.

\section{Data Extraction}

The data from all included studies were extracted into an electronic summary table by the first author (FT), which was then checked by another author (SA) after an independent PubMed and Web of Science search. Information collected related to the sample characteristics, study design, and relevant findings. The articles were then thematically arranged, and the findings reported accordingly. 


\section{RESULTS}

\section{Screening, inclusion, and exclusion of articles}

The search on PubMed and Web of Science identified 119 articles. Only sixteen articles fulfilled the inclusion criteria for the present systematic review, with only two being studies of rigorous design such as RCTs. Altogether, these sixteen articles reported data and study results derived from 3,792 participants. An overview of the search results is shown in Figure 1. The obtained articles reported studies on various themes, including investigating the effects of music on eating behavior, anxiety, mood and body perception in patients with or at risk of $\mathrm{AN}$ and $\mathrm{BN}$. None of the articles reported on the use of music or MT for BED. The design of the studies and their main results are depicted in Table 2. In this table, we have categorized these studies into three groups depending on whether music was used as active MT, passive MT or used as music videos on television and social media. In this results section, however, we summarize the studies according to the effect of music they report.

\section{Influence of music on eating behavior and food intake}

Cardi at al. investigated the impact of listening to music on the amount of food intake in patients with AN. Patients listened to four pieces of modern classical music by the Italian composer Ludovico Einaudi. Food consumption, psychological distress, desire to eat and attention bias to food were measured. This trial included both AN inpatients and outpatients (Cardi et al. 2013). The control group was exposed to a "vodcast", made of a mixture of visual images (e.g. landscapes) and unspecified soothing music. Inpatients who listened to piano music at mealtime consumed a higher quantity of the test meal compared to controls. Interestingly, these results were not corroborated by the outpatient group. Outpatients ate less of the test meal when exposed to music compared to the vodcast group.

In a different RCT investigating the association between mood and ED symptomatic behavior, Cardi et al. compared the effects of a "positive mood vodcast" (interventional group) against a "neutral vodcast" (control group) on the amount of a test meal consumed, attention bias to food and anxiety in 42 patients with ED (19 of these patients diagnosed with AN and 23 with BN) (Cardi et al. 2015). The "positive mood vodcast” had both visual (e.g. landscapes) and auditory (Mozart's Toy Symphony) mood-boosting components. The "neutral vodcast" was made of a static blue background, accompanied by the fast-paced instrumental Holst's Planets. They were played in the intervention and control group, respectively, whilst patients were having a test meal (fruit smoothie). The results showed a significant increase in meal consumption when listening to the positive mood vodcast in patients with AN, but no significant difference in meal consumption for the group with BN.

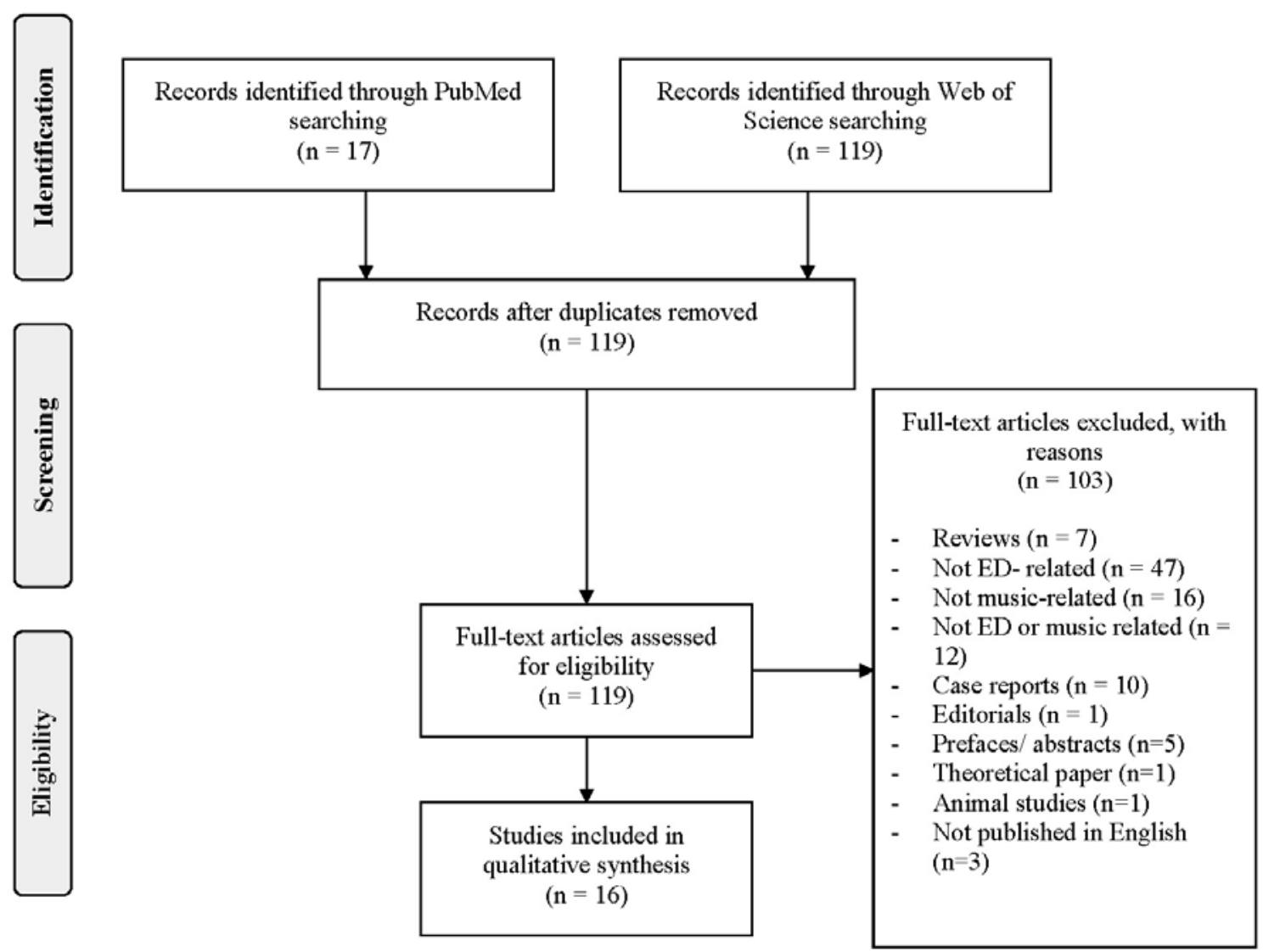

Figure 1. Overview of literature search 


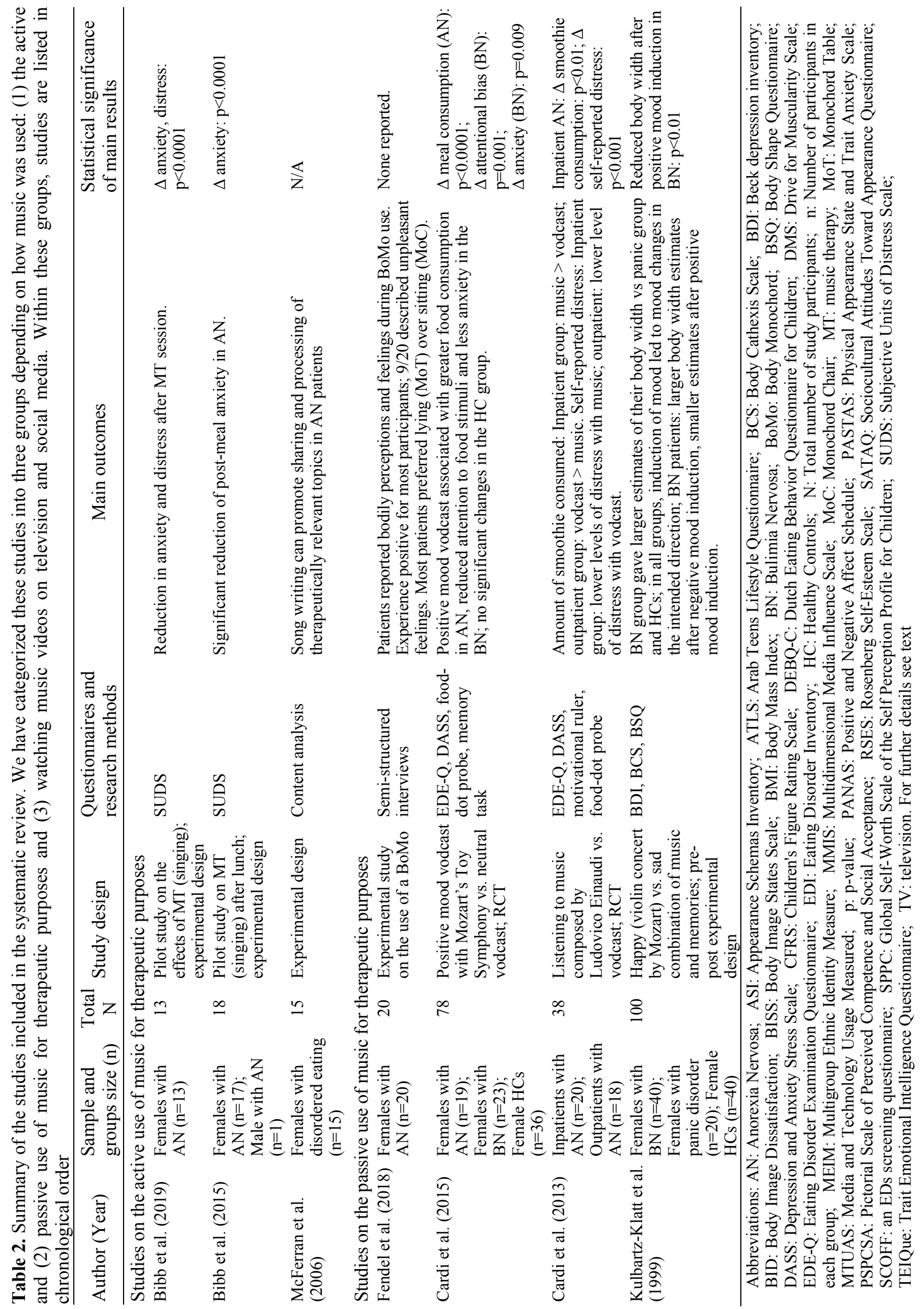




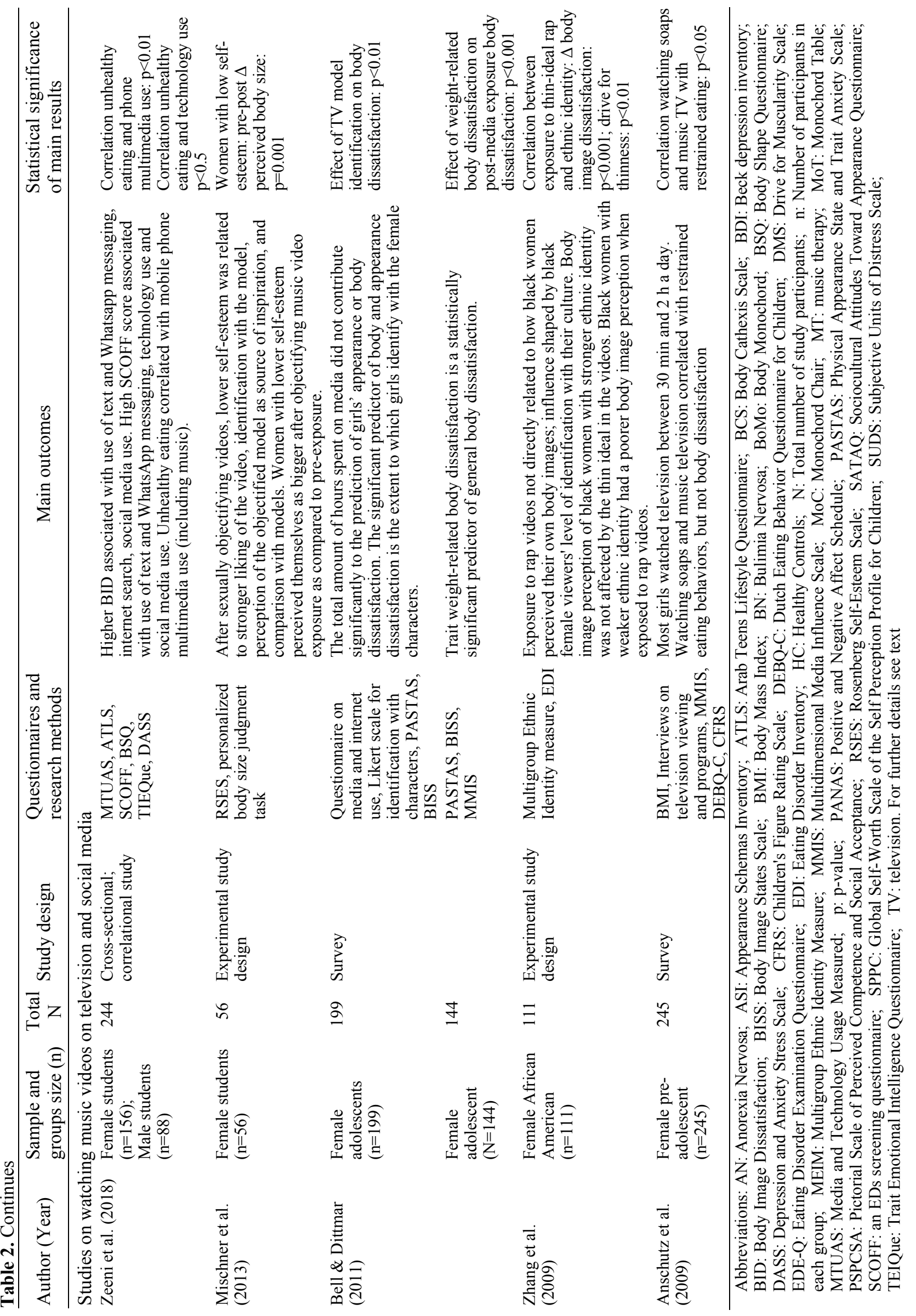




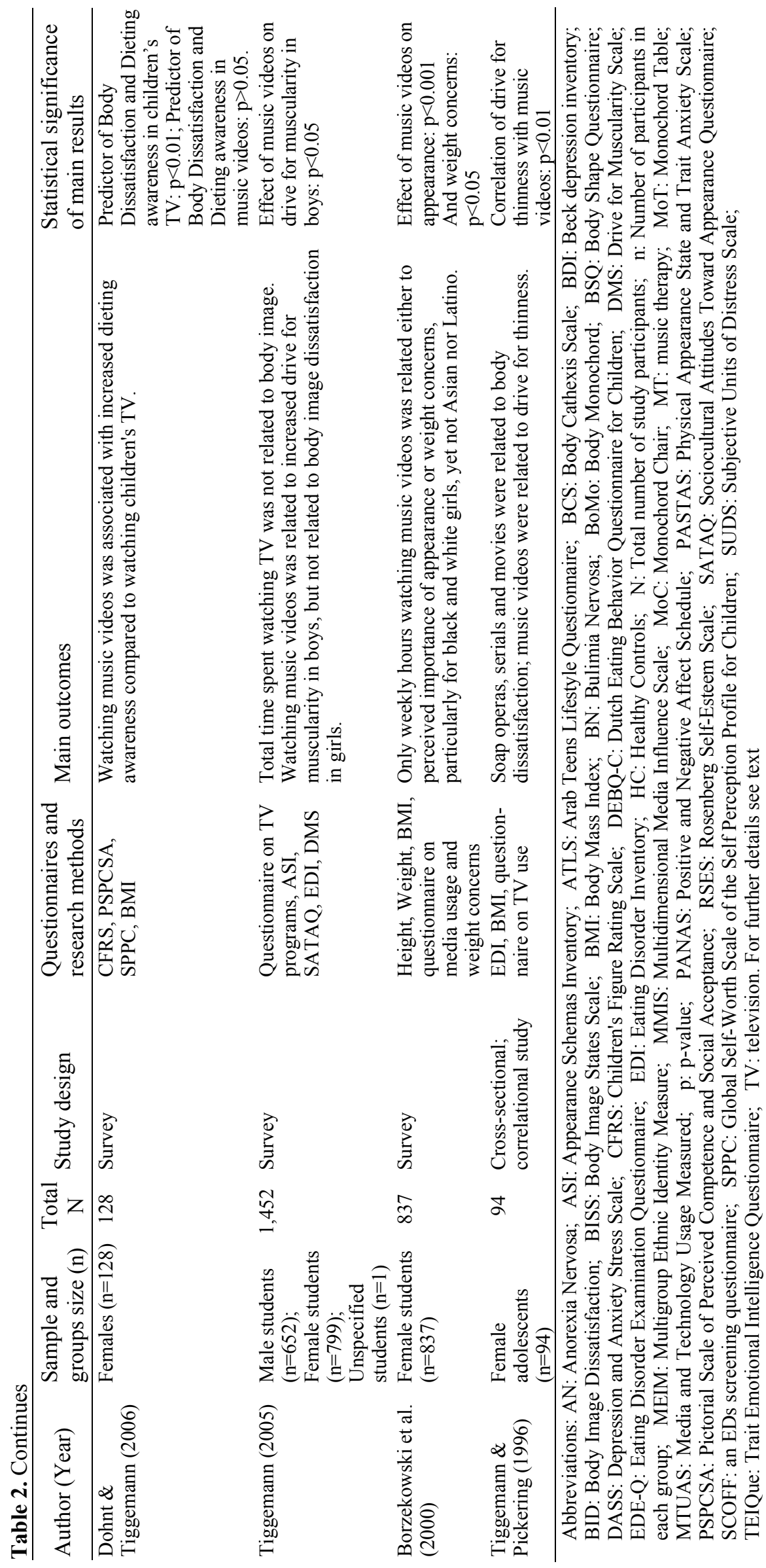




\section{Influence of music on anxiety, attention bias, processing of therapeutic issues and bodily experiences}

Five of the sixteen studies included analyzed the impact of music or MT on post-prandial anxiety, mealtime psychological distress, processing of therapeutic issues and bodily sensations.

Bibb et al. showed that a MT session after lunchtime significantly lowered post-prandial anxiety in inpatients and outpatients with AN (Bibb et al. 2015, 2019), as well as pre-prandial anxiety in inpatients (Bibb et al. 2015). In both studies, MT sessions were led by a music therapist who encouraged patients to actively engage in the group through singing, listening to songs, sharing thoughts on music, and writing songs (Bibb et al. 2015, 2019). Whilst the efficacy of MT interventions on reducing postprandial anxiety in the inpatient group was compared to conventional therapeutic support (Bibb et al. 2015), there was no control group in the outpatient study (Bibb et al. 2019). Both studies lacked randomization, the sample sizes were small, and the population was female-predominant.

In the study of Cardi et al. on the impact of classical piano music on food consumption (Cardi et al. 2013), inpatients who listened to piano music at mealtime reported lower levels of distress and consumed a higher quantity of the test meal compared to controls (vodcast group). In Cardi et al.'s study on a "positive mood vodcast” using Mozart's Toy Symphony, a reduction in attention bias to food and anxiety was found in patients with BN when compared to the group exposed to the neutral one (Cardi et al. 2015). Despite drinking a larger volume of the smoothie, patients with AN did not report any significant change in their anxiety level or attention bias to food.

McFerran et al. carried out a retrospective qualitative analysis of songs written by adolescent inpatients with AN during one-to-one MT sessions (McFerran et al. 2006). Themes arising from the lyrics were categorized in the following six groups: relationship dynamics, aspirations, reference to the disorder and its impact, emotional awareness, accessing support and identity formation, with the latter being the most dominant theme (McFerran et al. 2006). In the study, songwriting is described as a valuable and effective tool in soliciting information often not shared with the multi-disciplinary team and ultimately aiding the processing of therapeutic issues (McFerran et al. 2006).

Fendel et al. investigated the use of a Body Monochord (BoMo) in evoking different bodily sensations in inpatients diagnosed with AN (Fendel et al. 2018). During the sessions, the musician played the BoMo located behind and in direct contact with either a Monochord Chair (MoC) or Monochord Table (MoT). Participants were asked to sit either on the MoC or MoT (Fendel et al. 2018). The experiences reported by patients were classified into differentiated perception, focused attention, the emergence of body-related feelings, the emergence of emotions, the emergence of thoughts, the emergence of inner images, relaxation, spatial and temporal experience, new bodily experiences and self-reflection. Sensations rarely occurred in most-commonly reported problematic areas (e.g. belly or thighs) and were independent of body shape and size. Whilst participation in the BoMo sessions elicited positive feelings in most of the patients, such as deep relaxation and a general sense of wellbeing, more than a third of the participants reported unpleasant and physically uncomfortable sensations (Fendel et al. 2018).

\section{Influence of music on body perception, body dissatisfaction, and eating behavior}

Kulbartz-Klatt et al. investigated the effect of uplifting music (Mozart's Concerto No. 5, for violin and orchestra) and happy autobiographical memory on body width perception and estimation in inpatients and outpatients with BN (Kulbartz-Klatt et al. 1999). The control group was exposed to a negative-mood induction method using personal memories and lessuplifting music (Liszt's Via Crucis). The study had a pre-post controlled experimental design. It included an additional sample of women with the clinical diagnosis of panic disorder and a control group without any psychological disorder.

Patients with BN who experienced the positive-mood induction combination gave a smaller estimation of their body width. When exposed to the negative mood induction method, they reported a larger estimation of their body width. These results were seen in the group with BN only, although all the participants reported a moodcongruent change with the chosen induction method.

Zeeni et al. investigated the role of multimedia use in physical and mental wellbeing, with a specific focus on factors predisposing to EDs (Zeeni et al. 2018). Whilst higher body image dissatisfaction and increased risk for EDs were associated with text messages, WhatsApp messages, and social media browsing (especially Facebook and Instagram), unhealthy eating patterns were seen with extensive use of phone multimedia only (music, video, pictures) (Zeeni et al. 2018).

Two independent studies on pre-adolescent populations correlated music video-watching with increased dieting awareness and restrained eating behavior, respectively (Dohnt \& Tiggemann 2006, Anschutz et al. 2009). Dohnt \& Tiggemann explored the role of peers and media in body image perception in young schoolgirls aged between 5 and 8 years. The study highlighted that girls who watched music television were significantly more aware of dieting compared to the participants who watched "children's television" (e.g. a selection of cartoons and infancy shows) (Dohnt \& Tiggemann 2006). Other strong predictors for increased dieting awareness include reading adult magazine and perceived peer body dissatisfaction (Dohnt \& Tiggemann 2006). Along similar lines, Anschutz et al. highlighted that exposure to music videos and soap operas in pre- 
adolescence was directly associated with restrained eating behaviors, and indirectly associated with body dissatisfaction through internalization of the "thin" ideal (Anschutz et al. 2009).

A study conducted by Tiggemann and Pickering found a significant correlation between music videowatching and body dissatisfaction and drive for thinness in healthy teenage girls with a low Body Mass Index (BMI) (Tiggemann \& Pickering 1996). Data showed that each of the programs considered - comedies, sports, soap operas, series, movies, and music videos - was associated with increased body dissatisfaction yet the drive for thinness was reported only upon watching music channels. Similarly, Borzekowski et al. highlighted the association between music video-watching and increased preoccupation for own body weight or perceived importance of physical appearance (Borzekowski et al. 2000). Interestingly, these results were predominantly seen in white, black, and Latino teenage girls, but not in Asian girls (Borzekowski et al. 2000), though the correlation between ethnicity and music video watching is not statistically significant. A study conducted by Bell \& Dittmar confirmed the association between music videos and body and general appearance dissatisfaction. However, this correlation was stronger in girls who identify themselves with the "thin" ideal (Bell \& Dittmar 2011). Despite these results, a more recent study by Tiggemann associated music video watching with the drive for muscularity in adolescent boys rather than with weight concerns in girls (Tiggemann 2005).

Two independent studies explored the effect of sexually objectifying music videos on body image perception (Mischner et al. 2013, Zhang et al. 2009). Mischner et al. found that watching music television with stereotypical and sexist attitudes altered perception of body image in young women with low self-esteem, but not in those with high self-esteem (Mischner et al. 2013). After exposure to the selected videos, not only did participants perceive themselves as bigger, they also reported an increased discrepancy between their perceived and ideal body size, compared to pre-exposure data (Mischner et al. 2013). A study conducted by Zhang et al. discovered that the strength of ethnic identity determined the impact of sexually objectifying rap videos on body image (Zhang et al. 2009). Findings showed black young women with weaker cultural identity reported increased body dissatisfaction, drive for thinness and bulimic tendencies when exposed to the thin-ideal and the female objectification of rap videos; whilst participants with a stronger ethnic identity were less affected by video-exposure (Zhang et al. 2009).

\section{DISCUSSION}

In summary, we found that music could be either beneficial or deleterious for people with or at risk for an ED. When used as a therapeutic adjuvant in patients with a diagnosed ED, findings were overall rather positive. Classical piano music was beneficial in improving food consumption and peri-prandial distress in inpatients, but not outpatients with AN (Cardi et al. 2013). A “positive vodcast” using Mozart's Toy Symphony changed eating behavior favorably in patients with AN, but not BN. It helped patients with $\mathrm{BN}$ with their anxiety, but not patients with AN (Cardi et al. 2015). Active participation in MT sessions reduced postprandial anxiety in inpatients and out-patients with AN (Bibb et al. 2015, 2019). Songwriting facilitated the processing of therapeutic issues in adolescent inpatients with AN (McFerran et al. 2006), and exposure to BoMo sessions elicited diverse bodily sensations in anorexic participants (Fendel et al. 2018). Exposure to music altered the estimation of body perception. Listening to a violin concert by Mozart with the induction of happy autobiographical memories resulted in the reduced estimation of body width in patients with BN (KulbartzKlatt et al. 1999). Watching music videos, however, was associated with increased body dissatisfaction and drive for thinness in teenage girls with low BMI (Tiggemann \& Pickering 1996), bodyweight concerns and increased preoccupation for physical appearance (Borzekowski et al. 2000), body dissatisfaction in girls who identified themselves with the "thin" ideal (Bell \& Dittmar 2011) and increased drive for muscularity in adolescent boys (Tiggemann 2005). Exposure to sexually objectifying music videos led to the increased estimation of body size in young women with low self-esteem (Mischner et al. 2013), and body dissatisfaction, drive for thinness and bulimic tendencies in black young women with weak ethnical identity (Zhang et al. 2009). In preadolescent girls, exposure to music television increased dieting awareness (Dohnt \& Tiggemann 2006) and promoted restrained eating behavior and idealization of the thin ideal (Anschutz et al. 2009). Body dissatisfaction and unhealthy eating patterns were also seen with the use of social media and phone multimedia (including videos and music) (Zeeni et al. 2018).

Findings from this systematic review highlight that the effect of music varies substantially according to the setting, diagnosis, type of music, personality traits (including self-esteem) and cultural identity. However, overall evidence from observational, experimental or clinical studies for the use of music in EDs is scarce and additional data is needed for more convincing results.

When used therapeutically in individuals with a diagnosis of an ED, music had an overall positive impact on symptomatology. In patients with AN, exposure to music improved peri-prandial and post-prandial anxiety, attention bias to food and food consumption. In these instances, a wide range of music interventions was used, such as active participation in MT sessions, passive listening to piano music and vodcasts. Whilst this indicates that exposure to MT is beneficial, the most effective approach is less clear.

Using the same therapeutic intervention for both AN and BN (Cardi et al. 2015) did not provide consistent results among the two groups. Findings on the efficacy of MT in inpatients and outpatients with AN also 
differed. Whilst active participation in MT sessions was equally beneficial in both groups and different disease severity, passive listening to piano music produced positive results in inpatients only. This suggests the need to consider different approaches for different ED diagnoses, and to tailor the intervention to the patient's treatment setting.

In patients with AN, MT appeared to be efficacious in addressing therapeutic issues and evoking bodily sensations, which are both difficult to target with standard psychological therapy. This suggests that the adjunct of music to routine treatment programs could facilitate the processing of complex psychological issues in EDs.

A significant finding from our analysis was the impact of music in television and social media on body perception on pediatric, adolescent and young adult populations. Overall, exposure to music videos promoted body dissatisfaction, unhealthy approach to eating and concerns for weight and body image. Protective factors for this were high self-esteem, lack of identification with the "thin" ideal, and strong ethnic background (in black women), suggesting that personality traits and cultural background might have a role in the onset and progression of EDs. This finding offers the opportunity to reflect on the role of society and the music industry in ED development.

On the other hand, exposure to uplifting music together with happy autobiographical memories had a positive impact on body image perception. This suggests that music may modulate a variable construct such as body image, with significant implications in the clinical setting.

In summary, exposure to music appeared to be beneficial in individuals with a diagnosis of an ED when used in a clinical setting. However, music was found to be potentially detrimental when consumed in the form of multimedia, social media, and music videos.

Although some results are promising, they must be interpreted with caution and considered in light of many limitations. As discussed, data on the experience or use of music in EDs is very limited, with only sixteen studies deemed eligible for this systematic review, with very variable sample sizes (see Table 2). Due to the different approaches used, there are no confirmatory studies available for any of the reported positive findings. Therefore, the reproducibility of the results has not been shown yet. Despite the limited number of rigorous RCTs, there are many case reports on the successful use of MT in ED (Heiderscheit 2016).

Twelve out of the sixteen selected studies involved passive participation in music with only one of those featuring music alone as an intervention. The other studies combined music with either some visual stimuli (e.g. projected images, videos) or recall of autobiographical memories. Therefore, it is difficult to ascertain whether the resulting data was attributable to music alone, to other components, or their combination.
The study of Kulbartz-Klatt et al. (1999) used a combination of instrumental music and reminiscence of autobiographical memories, either happy or sad, according to the type of mood which they intended to elicit. Similarly, Cardi et al. (2013) investigated the effect of "positive mood vodcasts" which included music but also positive visual stimuli. Again, all studies on the impact of music videos and multimedia included other components besides music. Thus, the specific contribution of music to the effect of these combinations cannot be ascertained. Furthermore, it is unclear from the manuscripts of the included RCTs, what study participants received as main therapy. To interpret the findings in-depth, information on additional psychotherapeutic, psychopharmacological and family therapy is needed.

Only three studies reviewed had participants who actively participated in MT through singing, writing songs, or discussing music-related topics within a group (Bibb et al. 2015, 2019, McFerran 2006). Instrument playing and other areas of performance were not addressed in the eligible manuscripts. It should also be noted that the investigated samples were female-predominant and only one study (Tiggemann 2005) offered an insight into gender differences in response to music.

\section{CONCLUSION}

As the results of the published studies (Anschut et al. 2009, Bibb et al. 2015, 2019, Borzekowsk et al. 2000, Cardi et al. 2013, 2015, Dohnt \& Tiggemann 2006, Fendel et al. 2018, Kulbartz-Klatt et al. 1999, Mc Ferran 2006, Mischner et al. 2013, Tiggemann 2005, Tiggemann \& Pickering 1996, Zeeni et al. 2018, Zhang et al. 2009) and this systematic review have shown, the use of music seems to be dependent on the study sample and the settings. Thus, a nuanced study design is warranted for future studies. The study design must predefine specific inclusion criteria (ED diagnosis, disease severity, the risk factors to develop an ED when risk populations are investigated), the setting (outpatient vs. inpatient, adult versus child and adolescent mental health services), additional therapeutic components and the outcomes. Sociodemographic and socioeconomic variables should also be documented and controlled, as these variables influence treatment outcome and mortality in patients with EDs (Himmerich et al. 2019a,b).

Outcomes could be eating behavior, body perception, self-esteem, distress or anxiety or any other symptom outlined in the diagnostic criteria of DSM-5 or ICD-10/ICD-11. They could also include other problems frequently encountered in this group of patients like mood, reduced sleep quality and impairment in social occupational, or other important areas of functioning. Studies should also consider patient-reported outcome measures (PROMs) and patient-reported experience measures (PREMs) as outcomes for RCTs. PROMs and PREMs assess the effectiveness, safety, and experience of care from patients' perspective (Petit- 
Zeman et al. 2010) and seem to be of specific importance in the treatment of EDs (Himmerich et al. 2018).

In psychopharmacological trials, double blinding the patient and the treating physician is gold standard for RCTs. Unfortunately, this is not possible in psychotherapeutic or MT studies. However, a control group that would receive a different kind of support, management and randomization of study participants can be applied. These standards of clinical trials should be adhered to in studies on the use of music as a therapeutic element or MT to investigate the efficacy and effectiveness of such approaches.

The studies demonstrated that watching music videos on television or social media might have a negative impact on body dissatisfaction and the drive for thinness (Anschutz et al. 2009, Bell \& Dittmar 2011, Borzekowski et al. 2000, Dohnt \& Tiggemann 2006, Mischner et al. 2013, Tiggemann \& Pickering 1996, Tiggemann 2005, Zeeni et al. 2018, Zhang et al. 2009). This opens the research perspective on music in the context of television and social media. It also indicates that music could have side effects as highlighted in the study conducted by Fendel et al. (2018), just like psychotherapy could have unwanted effects (Linden 2013). Therefore, future studies should not only focus on the benefits but also the potential adverse effects of music on people with EDs.

\section{Acknowledgements:}

\section{Funding Statement}

Hubertus Himmerich has received salary support from the NIHR BRC at the South London and Maudsley NHS Foundation Trust (SLaM) and King's College London (KCL).

\section{Conflict of interest: None to declare.}

\section{Contribution of individual authors:}

Francesca Testa \& Hubertus Himmerich had the scientific idea for this systematic review and performed the literature search. The search was independently reproduced by Sarah Arunachalam.

Francesca Testa, Annie Heiderscheit \& Hubertus Himmerich wrote the first draft of the manuscript.

Francesca Testa \&, Sarah Arunachalam created the tables.

All authors discussed the manuscript and approved the final submitted version.

\section{References}

1. Aalbers $S$, Fusar-Poli L, Freeman RE, Spreen M, Ket JCF, Vink AC, et al.: Music therapy for depression. Cochrane Database Syst Rev 2017; 11:CD004517

2. American Psychiatric Association: Diagnostic and statistical manual of mental disorders (5th ed.). Washington DC, 2013
3. Anschutz D, Engels $R$, Van Leeuwe $J$ and \& van Strien T: Watching your weight? The relations between watching soaps and music television and body dissatisfaction and restrained eating in young girls. Psychol Health 2009; 24:1035-1050

4. Bell BT \& Dittmar H: Does Media Type Matter? The Role of Identification in Adolescent Girls' Media Consumption and the Impact of Different Thin-Ideal Media on Body Image. Sex Roles: 2011; 65:478

5. Bibb J, Castle D \& Newton R: The role of music therapy in reducing post meal related anxiety for patients with anorexia nervosa. J Eat Disord 2015; 3:50

6. Bibb J, Castle D \& Skewes McFerran K: Reducing Anxiety through Music Therapy at an Outpatient Eating Disorder Recovery Service. J Creat Ment Health 2019, 14:306-314

7. Blinder BJ, Cumella EJ \& Sanathara VA: Psychiatric comorbidities of female inpatients with eating disorders. Psychosom Med 2006, 68: 454-62

8. Bodell L \& Keel P: Current treatment for anorexia nervosa: efficacy, safety, and adherence. Psychol Res Behav Manag 2010; 3:91-108

9. Borzekowski DLG, Robinson TND \& Killen JDK: Does the camera add 10 pounds? Media use, perceived importance of appearance, and weight concerns among teenage girls. J Adolesc Health 2000; 26:36-41

10. Cardi V, Esposito M, Clarke A, Schifano S, \& Treasure J: The impact of induced positive mood on symptomatic behaviour in eating disorders. An experimental, $A B / B A$ crossover design testing a multimodal presentation during a test-meal. Appetite 2015; 87:192-198

11. Cardi V, Lounes N, Kan C \& Treasure J: Meal support using mobile technology in Anorexia Nervosa. Contextual differences between inpatient and outpatient settings. Appetite 2013; 60:33-39

12. Darby A, Hay P, Mond J, Quirk F, Buttner P \& Kennedy $L$ : The rising prevalence of comorbid obesity and eating disorder behaviors from 1995 to 2005. Int J Eat Disord 2009; 42:104-108

13. Dohnt HK \& Tiggemann M: Body Image Concerns in Young Girls: The Role of Peers and Media Prior to Adolescence. $J$ Youth Adolescence 2006; 35:135

14. Eddy KT, Tabri N, Thomas JJ, Murray HB, Keshaviah A, Hastings $E$ et al.: Recovery from Anorexia Nervosa and Bulimia Nervosa at 22-Year Follow-Up. J Clin Psychiatry 2017; 78:184-189

15. Fendel U, Sandler H, Papachristou C, Voigt B, Rose $M \&$ Klapp BF: Bodily experiences of patients diagnosed with anorexia nervosa during treatment with the body monochord-A modified grounded theory approach. Arts Psychother 2018; 59:7-16

16. Guarda AS: Treatment of anorexia nervosa: insights and obstacles. Physiol Behav 2008; 94:113-120

17. Gutiérrez EOF \& Camarena VAT: Music therapy in generalised anxiety disorder. Arts Psychother 2015; 44:19-24

18. Heiderscheit A: Creative Arts Therapies and Clients with Eating Disorders. Jessica Kingsley Publishers, London/ Philadelphia, 2016

19. Heise S, Steinberg $H$ \& Himmerich $H$ : The discussion about the application and impact of music on depressive diseases throughout history and present. Fortschr Neurol Psychiatr 2013; 81:426-36

20. Herzog D, Sacks N, Keller M, Lavori P, Von Ranson K \& Gray H: Patterns and Predictors of Recovery in Anorexia 
Nervosa and Bulimia Nervosa. J Am Acad Child Adolesc Psychiatry 1993; 32:835-842

21. Himmerich $H$ \& Treasure J: Psychopharmacological advances in eating disorders. Expert Rev Clin Pharmacol 2018; 11:95-108

22. Himmerich $H$, Hotopf $M$, Shetty $H$, Schmidt U, Treasure J, Hayes $R$ et al.: Psychiatric comorbidity as a risk factor for mortality in people with anorexia nervosa. Eur Arch Psychiatry Clin Neurosci 2019a; 269:351-359

23. Himmerich H, Hotopf M, Shetty H, Schmidt U, Treasure J, Hayes $R$ et al.: Psychiatric comorbidity as a risk factor for the mortality of people with bulimia nervosa. Soc Psychiatry Psychiatr Epidemiol 2019b; 54:813-821

24. Himmerich H, Joaquim M, Bentley J, Kan C, Dornik J, Treasure $J$ et al.: Psychopharmacological options for adult patients with anorexia nervosa: the patients' and carers' perspectives. CNS Spectr 2018; 23:251-252

25. Keel P \& Mitchell J: Outcome in bulimia nervosa. Am J Psychiatry 1997; 154:313-321

26. Keski-Rahkonen A \& Mustelin L: Epidemiology of eating disorders in Europe: prevalence, incidence, comorbidity, course, consequences, and risk factors. Curr Opin Psychiatry 2016; 29:340-345

27. Kulbartz-Klatt Y, Florin I \& Pook M: Bulimia nervosa: Mood changes do have an impact on body width estimation. Br J Clin Psychol 1999; 38:279-287

28. Linardon J, Fairburn CG, Fitzsimmons-Craft EE, Wilfley $D E$ \& Brennan $L:$ The empirical status of the third-wave behaviour therapies for the treatment of eating disorders: A systematic review. Clin Psychol Rev 2017; 58:125-140

29. Linden M: How to define, find and classify side effects in psychotherapy: from unwanted events to adverse treatment reactions. Clin Psychol Psychother 2013; 20:286-296

30. McFerran K, Baker F, Patton GC \& Sawyer SM: A Retrospective Lyrical Analysis of Songs Written by Adolescents with Anorexia Nervosa. Eur Eat Disorders Rev 2006; 14:397-403

31. Micali N, Hagberg K, Petersen I \& Treasure J: The incidence of eating disorders in the UK in 2000-2009: findings from the General Practice Research Database. BMJ Open 2013; 3(5)

32. Milos $G$, Spindler A, Schnyder $U$ \& Fairburn $C$ : Instability of eating disorder diagnoses: prospective study. Br J Psychiatry 2005; 187:573-578

33. Mischner IHS, van Schie HT, Wigboldus DHJ, van Baaren RB \& Engels RCME: Thinking big: The effect of sexually objectifying music videos on bodily self-perception in young women. Body Image 2013; 10:26-34

34. Moher D, Liberati A, Tetzlaff J, Altman DG \& PRISMA Group: Preferred reporting items for systematic reviews and meta-analyses: The PRISMA statement. Open Med 2009; 3:123-130
35. National Institute for Health and Care Excellence: Eating Disorders: Recognition and Treatment (NICE Guidelines 69), 2017. Retrieved on 30/01/2020 from

https://www.nice.org.uk/guidance/ng69

36. Petit-Zeman S, Firkins L \& Scadding J: The James Lind Alliance: tackling research mismatches. Lancet 2010; 376:667-669

37. Rushing J, Jones L \& Carney C: Bulimia Nervosa: A Primary Care Review. Prim Care Companion J Clin Psychiatry 2003; 5:217-224

38. Schmidt U, Adan R, Böhm I, Campbell IC, Dingemans A, Ehrlich $S$ et al.: Eating disorders: the big issue. Lancet Psychiatry 2016; 3:313-315

39. Shiranibidabadi D \& Mehryar A: Music therapy as an adjunct to standard treatment for obsessive compulsive disorder and co-morbid anxiety and depression: A randomized clinical trial. J Affect Disord 2015; 184:13-17

40. Slade E, Keeney E, Mavranezouli I, Dias S, Fou L, Stockton $S$ et al. Treatments for bulimia nervosa: a network meta-analysis. Psychol Med 2018; 48:2629-2636

41. Smink FR, van Hoeken D \& Hoek HW: Epidemiology of Eating Disorders: Incidence, Prevalence and Mortality Rates. Curr Psychiatry Rep 2012; 14:406-414

42. Spindler A \& Milos G: Links between eating disorder symptoms severity and psychiatric comorbidity. Eat Behav 2007; 8:364-73

43. Steinhausen HC: Outcome of Eating Disorders. Child Adolesc Psychiatr Clin N Am 2009; 18:225-242

44. Tiggemann M: Television and adolescent body image: the role of program content and viewing motivation. $J$ Soc Clin Psychol 2005; 24:361-381

45. Tiggemann $M$ \& Pickering A: Role of television in adolescent women's body dissatisfaction and drive for thinness. Int J Eat Disord 1996; 20:199-203

46. Vocks S, Tuschen-Caffier B, Pietrowsky R, Rustenbach S, Kersting A \& Herpetz S: Meta analysis of the effectiveness of psychological and pharmacological treatments for binge eating disorder. Int $J$ Eat Disord 2010; 43:205-217

47. World Health Organization: The ICD-10 Classification of Mental and Behavioural Disorders: Clinical Descriptions and Diagnostic Guidelines. Geneva, 1992

48. Yinger OS \& Gooding L: Music Therapy and Music Medicine for Children and Adolescents. Child Adolesc Psychiatr Clin N Am 2014; 23:535-553

49. Zeeni N, Doumit R, Abi Kharma J \& Sanchez-Ruiz MJ: Media, Technology Use, and Attitudes: Associations With Physical and Mental Well-Being in Youth With Implications for Evidence-Based Practice. Worldviews Evid Based Nurs 2018; 15:304-312

50. Zhang Y, Dixon T \& Conrad K: Rap Music Videos and African American Women's Body Image: The Moderating Role of Ethnic Identity. J Commun 2009; 59:262-278

Correspondence:

Hubertus Himmerich, MD

Department of Psychological Medicine, King's College London

103 Denmark Hill, London SE5 8AF, UK

E-mail: hubertus.himmerich@kcl.ac.uk 